\title{
OPTIMAL STOPPING PROBLEMS IN DIFFUSION-TYPE MODELS WITH RUNNING MAXIMA AND DRAWDOWNS
}

\author{
PAVEL V. GAPEEV * AND \\ NEOFYTOS RODOSTHENOUS, ${ }^{* * * * *}$ London School of Economics
}

\begin{abstract}
We study optimal stopping problems related to the pricing of perpetual American options in an extension of the Black-Merton-Scholes model in which the dividend and volatility rates of the underlying risky asset depend on the running values of its maximum and maximum drawdown. The optimal stopping times of the exercise are shown to be the first times at which the price of the underlying asset exits some regions restricted by certain boundaries depending on the running values of the associated maximum and maximum drawdown processes. We obtain closed-form solutions to the equivalent free-boundary problems for the value functions with smooth fit at the optimal stopping boundaries and normal reflection at the edges of the state space of the resulting three-dimensional Markov process. We derive first-order nonlinear ordinary differential equations for the optimal exercise boundaries of the perpetual American standard options.

Keywords: Multidimensional optimal stopping problem; Brownian motion; running maximum and running maximum drawdown process; free-boundary problem; instantaneous stopping and smooth fit; normal reflection; change-of-variable formula with local time on surfaces; perpetual American option
\end{abstract}

2010 Mathematics Subject Classification: Primary 60G40; 34K10; 91B70

Secondary 60J60; 34L30; 91B25

\section{Introduction}

The main aim of this paper is to present closed-form solutions to the discounted optimal stopping problem of (4) for the initial process $X$ in a diffusion-type model containing its running maximum process $S$ and its running maximum drawdown process $Y$ defined in (1)-(3). This problem is related to the option pricing theory in mathematical finance, where the process $X$ can describe the price of a risky asset (e.g. a stock) on a financial market. The value of (4) can therefore be interpreted as the rational (or no-arbitrage) price of a standard perpetual American call or put option in a diffusion-type extension of the Black-Merton-Scholes model (see, e.g. [30, Chapter VIII, Section 2a], [26, Chapter VII, Section 25], and [4] for an extensive overview of other related results in the area).

Optimal stopping problems for running maxima of some diffusion processes given linear costs were studied by Jacka [15], Dubins et al. [5], and Graversen and Peskir [11], [12], among others, with the aim of determining the best constants in the corresponding maximal inequalities. A complete solution of a general version of the same problem was obtained

Received 24 July 2013; revision received 27 October 2013.

* Postal address: London School of Economics, Department of Mathematics, Houghton Street,

London WC2A 2AE, UK. Email address: p.v.gapeev@1se.ac.uk

** Current address: University of Leeds, School of Mathematics, Leeds LS2 9JT, UK.

Email address: n.rodosthenous@leeds.ac.uk

*** Supported by a scholarship from the Alexander S. Onassis Public Benefit Foundation. 
by Peskir [22], by means of the established maximality principle, which is equivalent to the superharmonic characterization of the value function. Discounted optimal stopping problems for certain payoff functions depending on the running maxima of geometric Brownian motions were initiated by Shepp and Shiryaev [28], [29], and then considered by Pedersen [21] and Guo and Shepp [13], among others, with the aim of computing rational values of perpetual American lookback (Russian) options. More recently, Guo and Zervos [14] derived solutions for discounted optimal stopping problems related to the pricing of perpetual American options with certain payoff functions depending on the running values of both the initial diffusion process and its associated maximum. Glover et al. [10] provided solutions of optimal stopping problems for integrals of functions depending on the running values of both the initial diffusion process and its associated minimum. The main feature of the resulting optimal stopping problems is that the normal reflection condition holds for the value function at the diagonal of the state space of the two-dimensional continuous Markov process having the initial process and its running extremum as the components, which implies the characterization of the optimal boundaries as extremal solutions of one-dimensional first-order nonlinear ordinary differential equations.

Asmussen et al. [1] considered perpetual American options with payoffs depending on the running maximum of some Lévy processes with two-sided jumps having phase-type distributions in both directions. Avram et al. [2] studied exit problems for spectrally negative Lévy processes and applied the results to solving optimal stopping problems for payoff functions depending on the running values of the initial processes or their associated maxima. Optimal stopping games with payoff functions of such type were considered by Baurdoux and Kyprianou [3], within the framework of models based on spectrally negative Lévy processes. Other complicated optimal stopping problems for the running maxima were considered in [9] for a jump-diffusion model with compound Poisson processes with exponentially distributed jumps, and in [19] (see also [20]) for a model based on spectrally negative Lévy processes. More recently, Peskir [24], [25] studied optimal stopping problems for three-dimensional Markov processes having the initial diffusion process as well as its maximum and minimum as the state space components. It was shown that the optimal boundary surfaces depending on the maximum and minimum of the initial process provide the maximal and minimal solutions of the associated systems of first-order nonlinear partial differential equations.

In this paper we obtain closed-form solutions to the perpetual American standard options pricing problem in an extension of the Black-Merton-Scholes model with path-dependent coefficients. The underlying asset price dynamics are described by a geometric diffusion-type process $X$ with local drift and diffusion coefficients, which essentially depend on the running values of the maximum process $S$ and the maximum drawdown process $Y$. It is shown that the optimal exercise times are the first times at which the process $X$ exits some regions restricted by certain boundaries depending on the running values of $S$ and $Y$. The process $Y$ represents the maximum of the difference between the running values of the underlying asset price and its maximum, and can therefore be interpreted as the maximum of the market depth. We derive closedform expressions for the value functions as solutions of the equivalent free-boundary problems and apply the maximality principle from [22] to describe the optimal boundary surfaces as the maximal solutions of first-order nonlinear ordinary differential equations. The starting points for these surfaces at the edges of the three-dimensional state space of $(X, S, Y)$ are specified from the solutions of the corresponding optimal stopping problem for the two-dimensional Markov process $(X, S)$ in a corresponding model in which the coefficients of the process $X$ depend only on the running maximum process $S$. The Laplace transforms of the drawdown process and other related characteristics associated with certain classes of the initial processes, 
such as some diffusion models and spectrally positive and negative Lévy processes, were studied by Pospisil et al. [27] and Mijatovic and Pistorius [18], respectively. Diffusion-type processes with given joint laws for the terminal level and supremum at an independent exponential time were constructed by Forde [7], by allowing the diffusion coefficient to depend on the running value of the initial process and its running minimum. Other important characteristics for such diffusion-type processes were recently derived by Forde et al. [8].

The paper is organized as follows. In Section 2 we formulate the associated optimal stopping problems for a necessarily three-dimensional continuous Markov process, which has the underlying asset price and the running values of its maximum and maximum drawdown as the state space components. The resulting optimal stopping problems are reduced to their equivalent free-boundary problems for the value functions, which satisfy the smooth-fit conditions at the stopping boundaries and the normal reflection conditions at the edges of the state space of the three-dimensional process. In Section 3 we obtain closed-form solutions of the associated free-boundary problems in which the sought boundaries are found as either maximal or unique solutions of first-order nonlinear ordinary differential equations, where we specify the starting values for the latter at the edges of the three-dimensional state space. In Section 4, by applying the change-of-variable formula with local time on surfaces, we verify that the resulting solutions of the free-boundary problems provide the expressions for the value functions and the optimal stopping boundaries for the underlying asset price process in the initial problem. The main results of the paper are stated in Theorems 1 and 2 .

\section{Preliminaries}

In this section we introduce the setting and notation of the three-dimensional optimal stopping problems which are related to the pricing of perpetual American standard options and formulate the equivalent free-boundary problems.

\subsection{Formulation of the problem}

For a precise formulation of the problem, let us consider a probability space $(\Omega, \mathcal{F}, \mathbb{P})$ with a standard Brownian motion $B=\left(B_{t}\right)_{t \geq 0}$. Assume that there exists a process $X=\left(X_{t}\right)_{t \geq 0}$ given by

$$
X_{t}=x \exp \left(\int_{0}^{t}\left(r-\delta\left(S_{u}, Y_{u}\right)-\frac{\sigma^{2}\left(S_{u}, Y_{u}\right)}{2}\right) \mathrm{d} u+\int_{0}^{t} \sigma\left(S_{u}, Y_{u}\right) \mathrm{d} B_{u}\right),
$$

where $\delta(s, y)>0$ and $\sigma(s, y)>0$ are continuously differentiable bounded functions on $[0, \infty]^{2}$. It follows that the process $X$ solves the stochastic differential equation

$$
\mathrm{d} X_{t}=\left(r-\delta\left(S_{t}, Y_{t}\right)\right) X_{t} \mathrm{~d} t+\sigma\left(S_{t}, Y_{t}\right) X_{t} \mathrm{~d} B_{t}, \quad X_{0}=x,
$$

where $x>0$ is given and fixed. Here the associated with $X$ running maximum process $S=\left(S_{t}\right)_{t \geq 0}$ and the corresponding running maximum drawdown process $Y=\left(Y_{t}\right)_{t \geq 0}$ are defined by

$$
S_{t}=s \vee \max _{0 \leq u \leq t} X_{u} \quad \text { and } \quad Y_{t}=y \vee \max _{0 \leq u \leq t}\left(S_{u}-X_{u}\right)
$$

for arbitrary $0<s-y \leq x \leq s$, so that $X$ is a diffusion-type process representing a unique solution of the stochastic differential equation in (2) (see, e.g. [17, Chapter IV, Theorem 4.6]).

The main purpose of the present paper is to derive a closed-form solution to the optimal stopping problem for the time-homogeneous (strong) Markov process $(X, S, Y)=\left(X_{t}, S_{t}, Y_{t}\right)_{t \geq 0}$ 
given by

$$
V_{*}(x, s, y)=\sup _{\tau} \mathbb{E}_{x, s, y}\left[\mathrm{e}^{-r \tau} G\left(X_{\tau}\right)\right]
$$

for any $(x, s, y) \in E^{3}$, where the supremum is taken over all stopping times $\tau$ with respect to the natural filtration of $X$, and the payoff function is either $G(x)=(L-x)^{+}$or $G(x)=$ $(x-K)^{+}$for some given constants $0<L, K<\infty$. Here $\mathbb{E}_{x, s, y}$ denotes the expectation under the assumption that the (three-dimensional) process $(X, S, Y)$ defined in (1)-(3) starts at $(x, s, y) \in E^{3}$, where $E^{3}=\left\{(x, s, y) \in \mathbb{R}^{3} \mid 0<s-y \leq x \leq s\right\}$ is the state space of the process $(X, S, Y)$. We assume that the process $X$ describes the price of a risky asset on a financial market, where $r$ is the riskless interest rate, $\delta(s, y)$ is the dividend rate paid to the asset holders, and $\sigma(s, y)$ is the volatility rate. The dependence of the dividend and volatility rates on the past dynamics of the asset in the financial market is often used in practise, although it has not been well captured by local or stochastic dividend and volatility models studied in the literature. Moreover, a wide range of quantitative trading strategies are based on capturing the realised volatility, which increases in the cases of substantial changes in the asset prices, such as in the model of (1)-(2).

The value of (4) is then actually a rational (or no-arbitrage) price of a perpetual American put or call option with payoff function $G(x)=(L-x)^{+}$or $G(x)=(x-K)^{+}$, where the expectation is taken under the (unique) martingale measure (see, e.g. [30, Chapter VII, Section $3 g]$ ).

\subsection{The structure of the optimal stopping times}

It follows from the general theory of optimal stopping problems for Markov processes (see, e.g. [26, Chapter I, Section 2.2]) that the optimal stopping time in the problem of (4) is given by

$$
\tau_{*}=\inf \left\{t \geq 0 \mid V_{*}\left(X_{t}, S_{t}, Y_{t}\right)=G\left(X_{t}\right)\right\},
$$

so that, taking into account the structure of the payoff function $G(x)=(L-x)^{+}$or $G(x)=$ $(x-K)^{+}$in (4), we further assume that the optimal stopping time from (5) takes either the form

$$
\tau_{*}=\inf \left\{t \geq 0 \mid X_{t} \leq a_{*}\left(S_{t}, Y_{t}\right)\right\} \quad \text { or } \quad \tau_{*}=\inf \left\{t \geq 0 \mid X_{t} \geq b_{*}\left(S_{t}, Y_{t}\right)\right\}
$$

for some function $0<a_{*}(s, y)<L$ or $K<b_{*}(s, y)<\infty$ to be determined. This assumption means that the set

$$
C^{\prime}=\left\{(x, s, y) \in E^{3} \mid a_{*}(s, y)<s-y \text { or } s<b_{*}(s, y)\right\}
$$

belongs to the continuation region for the optimal stopping problem of (4) which is given by

$$
C_{*}=\left\{(x, s, y) \in E^{3} \mid a_{*}(s, y)<x \text { or } x<b_{*}(s, y)\right\},
$$

and the corresponding stopping region is the closure of the set

$$
D_{*}=\left\{(x, s, y) \in E^{3} \mid x<a_{*}(s, y) \text { or } b_{*}(s, y)<x\right\} .
$$

\subsection{The free-boundary problem}

By means of standard arguments based on the application of Itô's formula, it is shown that the infinitesimal operator $\mathbb{L}$ of the process $(X, S, Y)$ acts on a function $F(x, s, y)$ from the class $C^{2,1,1}$ on the interior of $E^{3}$ according to the rule

$$
(\mathbb{L} F)(x, s, y)=(r-\delta(s, y)) x \partial_{x} F(x, s, y)+\frac{\sigma^{2}(s, y)}{2} x^{2} \partial_{x x}^{2} F(x, s, y)
$$


for all $0<s-y<x<s$. It follows from the fact that both payoff functions $G(x)=(L-x)^{+}$ and $G(x)=(x-K)^{+}$are convex that the value function $V_{*}(x, s, y)$ is convex in the variable $x$, and, thus, it is continuous in $x$ on the interval $(0, \infty)$. In order to find analytic expressions for the unknown value function $V_{*}(x, s, y)$ from (4) and the unknown boundary $a_{*}(s, y)$ or $b_{*}(s, y)$ from $(6)$, let us build on the results of general theory of optimal stopping problems for Markov processes (see, e.g. [26, Chapter IV, Section 8]). We can reduce the optimal stopping problem of (4) to the equivalent free-boundary problem for $V_{*}(x, s, y)$ with $a_{*}(s, y)$ or $b_{*}(s, y)$ given by

$$
\begin{gathered}
(\mathbb{L} V)(x, s, y)=r V(x, s, y) \quad \text { for }(x, s, y) \in C, \\
\left.V(x, s, y)\right|_{x=a(s, y)+}=L-a(s, y) \quad \text { or }\left.V(x, s, y)\right|_{x=b(s, y)-}=b(s, y)-K, \\
V(x, s, y)=(L-x)^{+} \text {or } V(x, s, y)=(x-K)^{+} \text {for }(x, s, y) \in D, \\
V(x, s, y)>(L-x)^{+} \text {or } V(x, s, y)>(x-K)^{+} \text {for }(x, s, y) \in C, \\
(\mathbb{L} V)(x, s, y)<r V(x, s, y) \text { for }(x, s, y) \in D,
\end{gathered}
$$

where $C$ and $D$ are defined as $C_{*}$ and $D_{*}$ in (8) and (9) with $a(s, y)$ and $b(s, y)$ instead of $a_{*}(s, y)$ and $b_{*}(s, y)$, respectively, and the instantaneous-stopping conditions in (11) are satisfied when $s-y \leq a(s, y)$ or $b(s, y) \leq s$, respectively, for each $0<y<s$. Observe that the superharmonic characterisation of the value function (see [6] and [26, Chapter IV, Section 9]) implies that $V_{*}(x, s, y)$ is the smallest function satisfying (10)-(13) with the boundary $a_{*}(s, y)$ or $b_{*}(s, y)$. Moreover, we further assume that, for the left-hand system of (10)-(14), the smooth fit and normal reflection conditions

$$
\left.\partial_{x} V(x, s, y)\right|_{x=a(s, y)+}=-1 \quad \text { and }\left.\quad \partial_{s} V(x, s, y)\right|_{x=s-}=0
$$

hold when $s-y \leq a(s, y)<s$, and that, for the right-hand system of (10)-(14), the normal reflection and smooth fit conditions

$$
\left.\partial_{y} V(x, s, y)\right|_{x=(s-y)+}=0 \quad \text { and }\left.\quad \partial_{x} V(x, s, y)\right|_{x=b(s, y)-}=1
$$

hold when $s-y<b(s, y) \leq s$, for each $0<y<s$. Otherwise, the normal reflection conditions in the right-hand part of (15) and in the left-hand part of (16) hold when either $a(s, y)<s-y$ or $s<b(s, y)$, respectively, for each $0<y<s$. The conditions in (15)-(16) follow from both the structure of the problem and the state space $E^{3}$. For example, in the right-hand system of (10)-(14), the optimal stopping time takes the form of the right-hand part of (6) whenever $s-y<b(s, y) \leq s$. Therefore, for every fixed $0<y<s$, the process $X$ either decreases to the value $s-y$, and, thus, the value of $Y$ increases and the normal reflection condition in the left-hand part of (16) holds, or $X$ increases to the value $b_{*}(s, y)$ and the smooth fit condition in the right-hand part of (16) holds.

Note that, when $\delta(s, y)=\delta(s)$ and $\sigma(s, y)=\sigma(s)$ holds in (1)-(2), the value function $V_{*}(x, s, y)=U_{*}(x, s)$ together with the boundary $a_{*}(s, y)=g_{*}(s)$ or $b_{*}(s, y)=h_{*}(s)$ satisfy the left-hand or the right-hand system of (10)-(14), respectively. Moreover, the smooth fit and normal reflection conditions

$$
\left.\partial_{x} U(x, s)\right|_{x=g(s)+}=-1 \quad \text { and }\left.\quad \partial_{s} U(x, s)\right|_{x=s-}=0
$$

hold in addition to the left-hand system of (10)-(14) when $0<g(s)<s$, and the natural boundary and smooth fit conditions

$$
\left.U(x, s)\right|_{x=0+}=0 \quad \text { and }\left.\quad \partial_{x} U(x, s)\right|_{x=h(s)-}=1
$$


hold in addition to the right-hand system of (10)-(14) when $K<h(s) \leq s$, for each $s>0$. Otherwise, the normal reflection and natural boundary conditions in the right-hand part of (17) and in the left-hand part of (18) respectively hold for the latter system when $s<h(s)$ for each $s>0$.

\section{Solution of the free-boundary problem}

In this section we obtain closed-form expressions for the value functions $V_{*}(x, s, y)$ in (4) for the payoffs of standard put and call options, and derive explicit expressions and firstorder nonlinear ordinary differential equations for the optimal exercise boundaries $a_{*}(s, y)$ and $b_{*}(s, y)$ from (6), as solutions to the free-boundary problems (10)-(14) with (15) and (16), respectively.

\subsection{The general solution of the free-boundary problem}

We first observe that the general solution of the equation in (10) has the form

$$
V(x, s, y)=C_{1}(s, y) x^{\gamma_{1}(s, y)}+C_{2}(s, y) x^{\gamma_{2}(s, y)},
$$

where $C_{i}(s, y), i=1,2$, are some arbitrary continuously differentiable functions and $\gamma_{2}(s, y)<0<1<\gamma_{1}(s, y)$ are given by

$$
\gamma_{i}(s, y)=\frac{1}{2}-\frac{r-\delta(s, y)}{\sigma^{2}(s, y)}-(-1)^{i} \sqrt{\left(\frac{1}{2}-\frac{r-\delta(s, y)}{\sigma^{2}(s, y)}\right)^{2}+\frac{2 r}{\sigma^{2}(s, y)}}
$$

for all $0<y<s$. Hence, applying the instantaneous-stopping conditions from (11) to the function in (19), we conclude that the equalities

$$
\begin{aligned}
& C_{1}(s, y) a^{\gamma_{1}(s, y)}(s, y)+C_{2}(s, y) a^{\gamma_{2}(s, y)}(s, y)=L-a(s, y), \\
& C_{1}(s, y) b^{\gamma_{1}(s, y)}(s, y)+C_{2}(s, y) b^{\gamma_{2}(s, y)}(s, y)=b(s, y)-K
\end{aligned}
$$

hold when $s-y \leq a(s, y)$ and $b(s, y) \leq s$, respectively, for each $0<y<s$. Moreover, using the smooth fit conditions from the left-hand part of (15) and the right-hand part of (16), we find that the equalities

$$
\begin{gathered}
C_{1}(s, y) \gamma_{1}(s, y) a^{\gamma_{1}(s, y)}(s, y)+C_{2}(s, y) \gamma_{2}(s, y) a^{\gamma_{2}(s, y)}(s, y)=-a(s, y), \\
C_{1}(s, y) \gamma_{1}(s, y) b^{\gamma_{1}(s, y)}(s, y)+C_{2}(s, y) \gamma_{2}(s, y) b^{\gamma_{2}(s, y)}(s, y)=b(s, y)
\end{gathered}
$$

hold when $s-y \leq a(s, y)$ and $b(s, y) \leq s$, respectively, for each $0<y<s$. Finally, applying the normal reflection conditions from the right-hand part of (15) and the left-hand part of (16) to the function in (19), we conclude that the equalities

$$
\begin{gathered}
\sum_{i=1}^{2}\left(\partial_{s} C_{i}(s, y) s^{\gamma_{i}(s, y)}+C_{i}(s, y) \partial_{s} \gamma_{i}(s, y) s^{\gamma_{i}(s, y)} \ln s\right)=0, \\
\sum_{i=1}^{2}\left(\partial_{y} C_{i}(s, y)(s-y)^{\gamma_{i}(s, y)}+C_{i}(s, y) \partial_{y} \gamma_{i}(s, y)(s-y)^{\gamma_{i}(s, y)} \ln (s-y)\right)=0
\end{gathered}
$$

hold when either $a(s, y)<s$ or $s<b(s, y)$ and either $a(s, y)<s-y$ or $s-y<b(s, y)$, respectively, for each $0<y<s$. Here the partial derivatives $\partial_{s} \gamma_{i}(s, y)$ and $\partial_{y} \gamma_{i}(s, y)$ take the 
form

$$
\begin{aligned}
& \partial_{s} \gamma_{i}(s, y)=\phi(s, y)-(-1)^{i} \frac{\phi(s, y)\left(\gamma_{1}(s, y)-\gamma_{2}(s, y)\right) \sigma^{3}(s, y)-2 r \partial_{s} \sigma(s, y)}{\sigma^{2}(s, y) \sqrt{\left(\gamma_{1}(s, y)-\gamma_{2}(s, y)\right)^{2} \sigma^{2}(s, y)+2 r}}, \\
& \partial_{y} \gamma_{i}(s, y)=\psi(s, y)-(-1)^{i} \frac{\psi(s, y)\left(\gamma_{1}(s, y)-\gamma_{2}(s, y)\right) \sigma^{3}(s, y)-2 r \partial_{y} \sigma(s, y)}{\sigma^{2}(s, y) \sqrt{\left(\gamma_{1}(s, y)-\gamma_{2}(s, y)\right)^{2} \sigma^{2}(s, y)+2 r}},
\end{aligned}
$$

for $i=1,2$, and the functions $\phi(s, y)$ and $\psi(s, y)$ are defined by

$$
\begin{aligned}
& \phi(s, y)=\frac{\sigma(s, y) \partial_{s} \delta(s, y)+2(r-\delta(s, y)) \partial_{s} \sigma(s, y)}{\sigma^{3}(s, y)} \\
& \psi(s, y)=\frac{\sigma(s, y) \partial_{y} \delta(s, y)+2(r-\delta(s, y)) \partial_{y} \sigma(s, y)}{\sigma^{3}(s, y)}
\end{aligned}
$$

for $0<y<s$.

\subsection{The solution to the problem in the two-dimensional $(X, S)$ setting}

We begin with the case in which $\delta(s, y)=\delta(s)$ and $\sigma(s, y)=\sigma(s)$ holds in (1)-(2), and, thus, we can define the functions $\beta_{i}(s)=\gamma_{i}(s, y), i=1,2$, as in (20). Then the general solution $V(x, s, y)=U(x, s)$ of the equation in (10) has the form of (19) with $C_{i}(s, y)=D_{i}(s)$ and $\gamma_{i}(s, y)=\beta_{i}(s)$ for $i=1,2$, and the stopping time takes the form of (6) with either the boundary $a_{*}(s, y)=g_{*}(s)$ or $b_{*}(s, y)=h_{*}(s)$, respectively. We further denote the state space of the two-dimensional (strong) Markov process $(X, S)$ by $E^{2}=\left\{(x, s) \in \mathbb{R}^{2} \mid 0<x \leq s\right\}$ and its diagonal by $d^{2}=\left\{(x, s) \in \mathbb{R}^{2} \mid 0<x=s\right\}$, as well as recall that the second component of $(X, S)$ can only increase at $d^{2}$, that is, when $X_{t}=S_{t}$ for $t \geq 0$.

3.2.1. The call option case. Let us first consider the payoff function $G(x)=(x-K)^{+}$in (4). In this case, taking into account the fact that $\beta_{2}(s)<0<1<\beta_{1}(s)$, we observe that $D_{2}(s)=0$ should hold in (19), since otherwise $U(x, s) \rightarrow \pm \infty$ as $x \downarrow 0$, which must be excluded, by virtue of the obvious fact that the value function in (4) is bounded at 0 , due to the natural boundary condition in the left-hand part of (18). Hence, solving the system of equations in (22) and (24) for the unknown function $C_{1}(s, y)=D_{1}(s)$ with $C_{2}(s, y)=D_{2}(s)=0$, we conclude that the function $V(x, s, y)=U(x, s)$ in (19) admits the representation

$$
U\left(x, s ; h_{*}(s)\right)=\frac{h_{*}(s)}{\beta_{1}(s)}\left(\frac{x}{h_{*}(s)}\right)^{\beta_{1}(s)} \quad \text { with } \quad h_{*}(s)=\frac{\beta_{1}(s) K}{\beta_{1}(s)-1}
$$

for $0<x<h_{*}(s) \leq s$, so that $h_{*}(s)>K \vee(r K / \delta(s))$ holds due to the facts that $\beta_{2}(s)<$ $0<1<\beta_{1}(s)$ in $(20)$ and $\beta_{1}(s) \beta_{2}(s) /\left(\left(\beta_{1}(s)-1\right)\left(\beta_{2}(s)-1\right)\right)=r / \delta(s)$ for all $s>K$.

In this case, we set $\widetilde{s}_{0}=\infty$ and define a decreasing sequence $\left(\widetilde{s}_{n}\right)_{n \in \mathbb{N}}$ such that the boundary $h_{*}(s)$ from (31) exits the region $E^{2}$ at $\left(\widetilde{s}_{2 l-1}, \widetilde{s}_{2 l-1}\right)$ and re-enters $E^{2}$ downwards at $\left(\widetilde{s}_{2 l}, \widetilde{s}_{2 l}\right)$. Namely, we define $\widetilde{s}_{2 l-1}=\sup \left\{s<\widetilde{s}_{2 l-2} \mid h_{*}(s)>s\right\}$ and $\widetilde{s}_{2 l}=\sup \left\{s<\widetilde{s}_{2 l-1} \mid h_{*}(s) \leq s\right\}$, $l \in \mathbb{N}$, whenever they exist, and put $\tilde{s}_{2 l-1}=\tilde{s}_{2 l}=0$ otherwise. Note that $K<\widetilde{s}_{2 l}<\widetilde{s}_{2 l-1}<$ $\infty, l \in \mathbb{N}$, by construction. Then the candidate value function admits the representation of (31) in the regions

$$
\widetilde{R}_{2 l-1}^{2}=\left\{(x, s) \in E^{2} \mid \widetilde{s}_{2 l-1}<s \leq \widetilde{s}_{2 l-2}\right\}
$$

for $l \in \mathbb{N}$ (see Figure 1). 


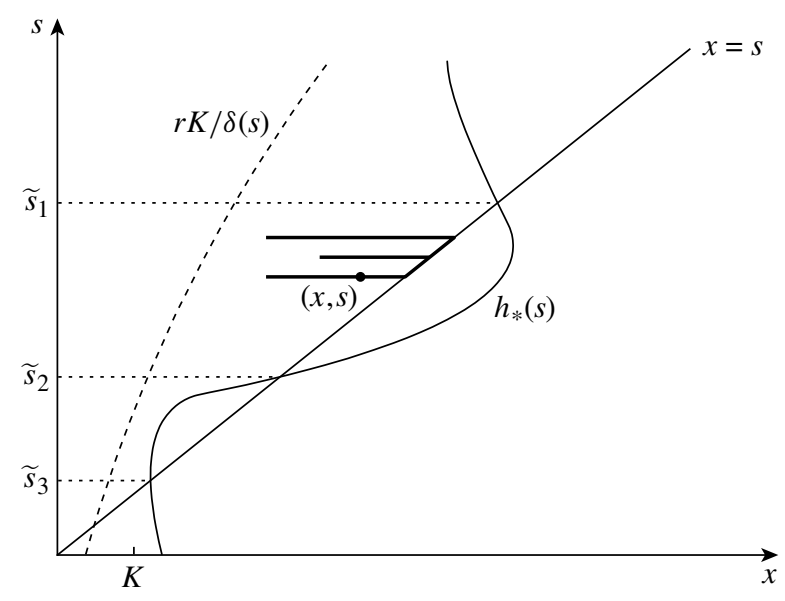

Figure 1: A computer drawing of the state space of the process $(X, S)$ and the boundary function $h_{*}(s)$.

On the other hand, the candidate value function $V(x, s, y)=U(x, s)$ takes the form of (19) with $C_{1}(s, y)=D_{1}(s)$ solving the first-order linear ordinary differential equation in (25) and $C_{2}(s, y)=D_{2}(s)=0$, in the regions

$$
\widetilde{R}_{2 l}^{2}=\left\{(x, s) \in E^{2} \mid \widetilde{s}_{2 l}<s \leq \widetilde{s}_{2 l-1}\right\}
$$

for $l \in \mathbb{N}$, which belong to $C^{\prime}$ in (7). Note that the process $(X, S)$ can pass from the region $\widetilde{R}_{2 l}^{2}$ in (33) to the region $\widetilde{R}_{2 l-1}^{2}$ in (32) only through the point $\left(\widetilde{s}_{2 l-1}, \widetilde{s}_{2 l-1}\right)$, for $l \in \mathbb{N}$. Thus, the candidate value function should be continuous at the point $\left(\widetilde{s}_{2 l-1}, \widetilde{s}_{2 l-1}\right)$, which is expressed by the equality

$$
D_{1}\left(\widetilde{s}_{2 l-1}\right)\left(\widetilde{s}_{2 l-1}\right)^{\beta_{1}\left(\widetilde{s}_{2 l-1}\right)}=U\left(\widetilde{s}_{2 l-1}+, \widetilde{s}_{2 l-1}+; h_{*}\left(\widetilde{s}_{2 l-1}+\right)\right),
$$

where the right-hand side is given by (31). Hence, solving the first-order linear ordinary differential equation in (25) for the unknown function $C_{1}(s, y)=D_{1}(s)$ with $C_{2}(s, y)=$ $D_{2}(s)=0$ and using the condition of (34), we conclude that the candidate value function $V(x, s, y)=U(x, s)$ in (19) admits the expression

$$
U\left(x, s ; \widetilde{s}_{2 l-1}\right)=\exp \left(-\int_{s}^{\widetilde{s}_{2 l-1}} \beta_{1}^{\prime}(q) \ln q \mathrm{~d} q\right) \frac{\left(\widetilde{s}_{2 l-1}\right)^{1-\beta_{1}\left(\tilde{s}_{2 l-1}\right)}}{\beta_{1}\left(\widetilde{s}_{2 l-1}\right)} x^{\beta_{1}(s)}
$$

in the regions $\widetilde{R}_{2 l}^{2}$ given by (33) for $l \in \mathbb{N}$.

3.2.2. The put option case. Let us now consider the payoff function $G(x)=(L-x)^{+}$in (4). In this case, solving the system of equations in (21) and (23) for the unknown functions $C_{i}(s, y)=D_{i}(s), i=1,2$, we conclude that the function $V(x, s, y)=U(x, s)$ in (19) admits the representation

$$
U\left(x, s ; g_{*}(s)\right)=D_{1}\left(s ; g_{*}(s)\right) x^{\beta_{1}(s)}+D_{2}\left(s ; g_{*}(s)\right) x^{\beta_{2}(s)}
$$

for $0<g_{*}(s)<x \leq s$, with

$$
D_{i}\left(s ; g_{*}(s)\right)=\frac{\left(\beta_{3-i}(s)-1\right) g_{*}(s)-\beta_{3-i}(s) L}{\left(\beta_{i}(s)-\beta_{3-i}(s)\right) g_{*}(s)^{\beta_{i}(s)}}
$$


for all $s>0$ and $i=1,2$. Hence, assuming that the boundary function $g_{*}(s)$ is continuously differentiable, we apply condition (25) to the functions $C_{i}(s, y)=D_{i}\left(s ; g_{*}(s)\right), i=1,2$, in (37) and conclude that $g_{*}(s)$ solves the first-order nonlinear ordinary differential equation

$$
\begin{aligned}
g^{\prime}(s)=\sum_{i=1}^{2} & \frac{\left(\left(\beta_{3-i}(s)-1\right) g(s)-\beta_{3-i}(s)\right) g(s)}{\left(\beta_{i}(s)-1\right)\left(\beta_{3-i}(s)-1\right) g(s)-\beta_{i}(s) \beta_{3-i}(s) L} \\
& \times\left(\frac{1}{\beta_{3-i}(s)-\beta_{i}(s)}+\frac{(s / g(s))^{\beta_{i}(s)} \ln (s / g(s))}{(s / g(s))^{\beta_{i}(s)}-(s / g(s))^{\beta_{3-i}(s)}}\right) \beta_{i}^{\prime}(s),
\end{aligned}
$$

where the derivatives $\beta_{i}^{\prime}(s)=\partial_{s} \gamma_{i}(s, y), i=1,2$, are given by (27) with (29). Taking into account the fact that $\beta_{i}(s), i=1,2$, and the boundary $g_{*}(s)$ are continuously differentiable functions in the neighbourhood of $\infty$, we observe that the function in (36) should satisfy the property $U\left(x, s ; g_{*}(s)\right) \rightarrow U\left(x, \infty ; g_{*}(\infty)\right)$ as $s \rightarrow \infty$ for each $x>g_{*}(s)$. Thus, using the fact that $\beta_{2}(s)<0<1<\beta_{1}(s)$, we obtain the expressions

$$
U\left(x, \infty ; g_{*}(\infty)\right)=\frac{g_{*}(\infty)}{\beta_{2}(\infty)}\left(\frac{x}{g_{*}(\infty)}\right)^{\beta_{2}(\infty)} \text { and } \quad g_{*}(\infty)=\frac{\beta_{2}(\infty) L}{\beta_{2}(\infty)-1}
$$

for $x>g_{*}(\infty)$. The form of the function $U\left(x, \infty ; g_{*}(\infty)\right)$ and the boundary $g_{*}(\infty)$ in (39) follows from the fact that $U\left(x, \infty ; g_{*}(\infty)\right) \rightarrow \pm \infty$ should not hold as $x \rightarrow \infty$ since the value function in (4) is bounded at $\infty$. Observe that the expressions in (39) coincide with those of the value function in the corresponding continuation region and the exercise boundary of the perpetual American put option in the Black-Merton-Scholes model with constant coefficients (see, e.g. [30, Chapter VIII, Section 2a]).

Let us now consider the maximal solution $g_{*}(s)$ of the first-order ordinary differential equation in (38) with the starting value $g_{*}(\infty)$ from (39) as $s \uparrow \infty$ and such that this solution stays below the curve $x=L \wedge(r L / \delta(s))$. Then we put $\widehat{s}_{0}=\infty$ and define a decreasing sequence $\left(\widehat{s}_{n}\right)_{n \in \mathbb{N}}$ such that the solution $g_{*}(s)$ of the equation in (38) exits the region $E^{2}$ at the points $\left(\widehat{s}_{2 k-1}, \widehat{s}_{2 k-1}\right)$ and enters $E^{2}$ downwards at the points $\left(\widehat{s}_{2 k}, \widehat{s}_{2 k}\right)$. Namely, we define $\widehat{s}_{2 k-1}=\sup \left\{s \leq \widehat{s}_{2 k-2} \mid g_{*}(s)>s\right\}$ and $\widehat{s}_{2 k}=\sup \left\{s \leq \widehat{s}_{2 k-1} \mid g_{*}(s) \leq s\right\}, k \in \mathbb{N}$, whenever they exist, and put $\widehat{s}_{2 k}=\widehat{s}_{2 k-1}=0$ otherwise. Note that $0<\widehat{s}_{2 k}<\widehat{s}_{2 k-1}<L, k \in \mathbb{N}$, by construction. Then the candidate value function takes the form of (36)-(37) in the regions

$$
\widehat{Q}_{2 k-1}^{2}=\left\{(x, s) \in E^{2} \mid \widehat{s}_{2 k-1}<s \leq \widehat{s}_{2 k-2}\right\}
$$

for $k \in \mathbb{N}$, and the boundary function $g_{*}(s)$ provides the maximal solution of the equation in (38) started at $g_{*}(\infty)$ from (39) and such that it stays strictly below the curve $x=L \wedge(r L / \delta(s))$. Finally, we note that the candidate value function should be given by the condition in the left-hand part of (12) in the regions

$$
\widehat{Q}_{2 k}^{2}=\left\{(x, s) \in E^{2} \mid \widehat{s}_{2 k}<s \leq \widehat{s}_{2 k-1}\right\}
$$

for $k \in \mathbb{N}$, which belong to the stopping region $D_{*}$ in (9).

\subsection{The solution to the problem in the three-dimensional $(X, S, Y)$ setting}

We now continue with the general form of the coefficients $\delta(s, y)$ and $\sigma(s, y)$ in (1)-(2), and, thus, of the functions $\gamma_{i}(s, y), i=1,2$, from (20). We denote the border planes of the state space $E^{3}$ by $d_{1}^{3}=\left\{(x, s, y) \in \mathbb{R}^{3} \mid 0<x=s\right\}$ and $d_{2}^{3}=\left\{(x, s, y) \in \mathbb{R}^{3} \mid 0<x=s-y\right\}$, as well as recall that the second and third components of the process $(X, S, Y)$ can increase only at the planes $d_{1}^{3}$ and $d_{2}^{3}$, that is, when $X_{t}=S_{t}$ and $X_{t}=S_{t}-Y_{t}$ for $t \geq 0$, respectively. 
3.3.1. The call option case. Let us now return to the payoff function $G(x)=(x-K)^{+}$in (4). In this case, solving the system of equations in (22) and (24), we conclude that the function in (19) admits the representation

$$
V\left(x, s, y ; b_{*}(s, y)\right)=C_{1}\left(s, y ; b_{*}(s, y)\right) x^{\gamma_{1}(s, y)}+C_{2}\left(s, y ; b_{*}(s, y)\right) x^{\gamma_{2}(s, y)}
$$

for $0<s-y \leq x<b_{*}(s, y) \leq s$ and $s>K$, with

$$
C_{i}\left(s, y ; b_{*}(s, y)\right)=\frac{\left(\gamma_{3-i}(s, y)-1\right) b_{*}(s, y)-\gamma_{3-i}(s, y) K}{\left(\gamma_{3-i}(s, y)-\gamma_{i}(s, y)\right) b_{*}(s, y)^{\gamma_{i}(s, y)}}
$$

for all $0<y<s$ and $i=1,2$. Hence, assuming that the boundary function $b_{*}(s, y)$ is continuously differentiable, we apply condition (26) to the functions $C_{i}(s, y)=C_{i}\left(s, y ; b_{*}(s, y)\right)$, $i=1,2$, in (42) to conclude that $b_{*}(s, y)$ solves the first-order nonlinear ordinary differential equation

$$
\begin{aligned}
\partial_{y} b(s, y) & \\
=\sum_{i=1}^{2} & \frac{\left(\left(\gamma_{3-i}(s, y)-1\right) b(s, y)-\gamma_{3-i}(s, y) K\right) b(s, y)}{\left(\gamma_{i}(s, y)-1\right)\left(\gamma_{3-i}(s, y)-1\right) b(s, y)-\gamma_{i}(s, y) \gamma_{3-i}(s, y) K} \\
& \times\left(\frac{1}{\gamma_{3-i}(s, y)-\gamma_{i}(s, y)}+\frac{((s-y) / b(s, y))^{\gamma_{i}(s, y)} \ln ((s-y) / b(s, y))}{((s-y) / b(s, y))^{\gamma_{i}(s, y)}-((s-y) / b(s, y))^{\gamma_{3}-i}(s, y)}\right) \\
& \times \partial_{y} \gamma_{i}(s, y)
\end{aligned}
$$

for $0<y<s$, where the partial derivatives $\partial_{y} \gamma_{i}(s, y), i=1,2$, are given by (28) with (30).

Since the functions $\delta(s, y)$ and $\sigma(s, y)$ are assumed to be continuously differentiable and bounded, it follows that the limits $\delta(s, s-)$ and $\sigma(s, s-)$ exist for each $s>0$. Then the limits $\gamma_{i}(s, s-)$ can be identified with the functions $\beta_{i}(s), i=1,2$, from Subsection 3.2, and the function in (41) should satisfy the property $V\left(x, s, y ; b_{*}(s, y)\right) \rightarrow V\left(x, s, s-; b_{*}(s, s-)\right)$ as $y \uparrow s$ for each $s-y \leq x<b_{*}(s, y)$. Thus, taking into account the fact that $\gamma_{2}(s, y)<0<$ $1<\gamma_{1}(s, y)$, we conclude that the equalities

$$
V\left(x, s, s-; b_{*}(s, s-)\right)=U\left(x, s ; b_{*}(s, s-)\right) \quad \text { and } \quad b_{*}(s, s-)=h_{*}(s)
$$

hold for $0<x<b_{*}(s, s-)$ and $s>K$, with $U\left(x, s ; h_{*}(s)\right)$ and $h_{*}(s)$ given by (31), since otherwise $V\left(x, s, s-; b_{*}(s, s-)\right) \rightarrow \pm \infty$ as $x \downarrow 0$, which must be excluded by virtue of the obvious fact that the value function in (4) is bounded at 0 .

For any fixed $s>K$, let us now consider the solution $b_{*}(s, y)$ of (43) started from the value $h_{*}(s)$ given by (31) at $y \uparrow s$, given that this solution stays strictly above the surface $x=$ $K \vee(r K / \delta(s, y))$. Then we put $\tilde{y}_{0}(s)=s$ and define a decreasing sequence $\left(\tilde{y}_{n}(s)\right)_{n \in \mathbb{N}}$ such that $\tilde{y}_{2 l-1}(s)=\sup \left\{y<\tilde{y}_{2 l-2}(s) \mid b_{*}(s, y)>s\right\}$ and $\tilde{y}_{2 l}(s)=\sup \left\{y<\tilde{y}_{2 l-1}(s) \mid b_{*}(s, y) \leq s\right\}$, whenever they exist, and put $\tilde{y}_{2 l-1}(s)=\tilde{y}_{2 l}(s)=0, l \in \mathbb{N}$, otherwise. Moreover, we can also define a decreasing sequence $\left(\widehat{y}_{n}(s)\right)_{n \in \mathbb{N}}$ such that the boundary $b_{*}(s, y)$ exits the region $E^{3}$ from the side of $d_{2}^{3}$ at the points $\left(s-\widehat{y}_{2 k-1}(s), s, \widehat{y}_{2 k-1}(s)\right)$ and enters $E^{3}$ downwards at the points $\left(s-\widehat{y}_{2 k}(s), s, \widehat{y}_{2 k}(s)\right)$. Namely, we put $\widehat{y}_{0}(s)=s$ and define $\widehat{y}_{2 k-1}(s)=$ $\sup \left\{y<\widehat{y}_{2 k-2}(s) \mid b_{*}(s, y)<s-y\right\}$ and $\widehat{y}_{2 k}(s)=\sup \left\{y<\widehat{y}_{2 k-1}(s) \mid b_{*}(s, y) \geq s-y\right\}$ whenever such points exist, and put $\widehat{y}_{2 k-1}(s)=\widehat{y}_{2 k}(s)=0$ otherwise for $k \in \mathbb{N}$. Note that $0<\widehat{y}_{2 k}(s)<\widehat{y}_{2 k-1}(s)<s-K, k \in \mathbb{N}$, by construction. Therefore, the candidate value 


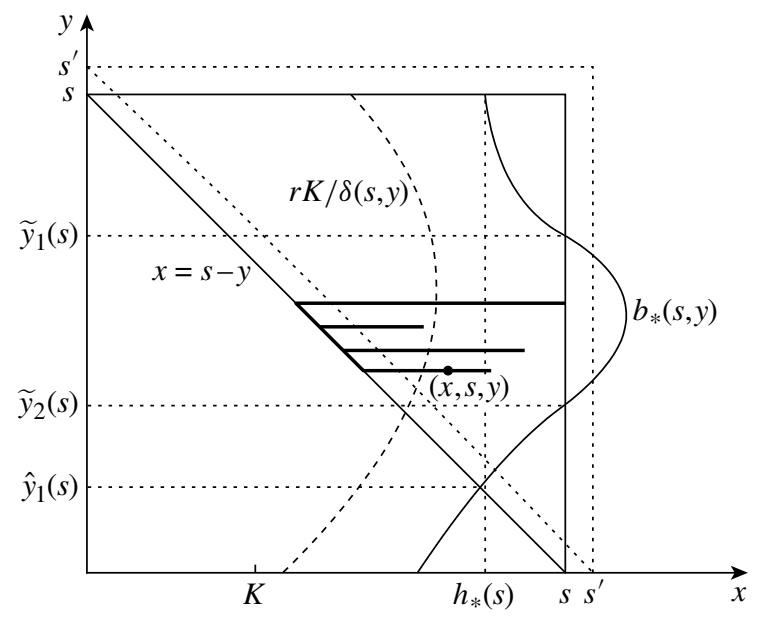

Figure 2: A computer drawing of the state space of the process $(X, S, Y)$ for some fixed $s$, which increases to $s^{\prime}$, and the boundary function $b_{*}(s, y)$.

function admits the expression in (41)-(42) in either the region

$$
\widetilde{R}_{2 l-1}^{3}=\left\{(x, s, y) \in E^{3} \mid \tilde{y}_{2 l-1}(s)<y \leq \min _{k \in \mathbb{N}}\left\{\widehat{y}_{2 k-2}(s) \mid \tilde{y}_{2 l-1}(s)<\widehat{y}_{2 k-2}(s)\right\} \wedge \tilde{y}_{2 l-2}(s)\right\}
$$

or

$\widehat{R}_{2 k-1}^{3}=\left\{(x, s, y) \in E^{3} \mid \widehat{y}_{2 k-1}(s)<y \leq \min _{l \in \mathbb{N}}\left\{\tilde{y}_{2 l-1}(s) \mid \tilde{y}_{2 l-1}(s)<\widehat{y}_{2 k-2}(s)\right\} \wedge \widehat{y}_{2 k-2}(s)\right\}$

for $k, l \in \mathbb{N}$, and the boundary $b_{*}(s, y)$ provides the unique solution of the equation in (43) started from the value $b_{*}(s, s-)=h_{*}(s)$ from (31) and such that this solution stays strictly above the surface $x=K \vee(r K / \delta(s, y))$ (see Figure 2).

On the other hand, the candidate value function takes the form of (19) with $C_{i}(s, y), i=1,2$, solving the linear system of first-order partial differential equations in (25) and (26) in the regions

$$
\widetilde{R}_{2 l}^{3}=\left\{(x, s, y) \in E^{3} \mid \tilde{y}_{2 l}(s)<y \leq \tilde{y}_{2 l-1}(s)\right\}
$$

for $l \in \mathbb{N}$, which belong to $C^{\prime}$ in (7). Note that the process $(X, S, Y)$ can enter the region $\widetilde{R}_{2 l}^{3}$ in (46) from one of the regions $\widetilde{R}_{2 l+1}^{3}$ in (44) or $\widehat{R}_{2 k-1}^{3}$ in (45) for some $k \in \mathbb{N}$ only through the point $\left(s-\tilde{y}_{2 l}(s), s, \tilde{y}_{2 l}(s)\right)$, and can exit the region $R_{2 l}^{3}$ passing to the region $\widetilde{R}_{2 l-1}^{3}$ only through the point $\left(s-\tilde{y}_{2 l-1}(s), s, \tilde{y}_{2 l-1}(s)\right)$ by hitting the plane $d_{2}^{3}$ and increasing its third component $Y$. Thus, the candidate function should be continuous at the points $\left(s-\tilde{y}_{2 l}(s), s, \tilde{y}_{2 l}(s)\right)$ and $\left(s-\tilde{y}_{2 l-1}(s), s, \tilde{y}_{2 l-1}(s)\right)$, which is expressed by the equalities

$$
\begin{aligned}
& C_{1}\left(s, \tilde{y}_{2 l}(s)+\right)\left(\left(s-\tilde{y}_{2 l}(s)\right)-\right)^{\gamma_{1}\left(s, \tilde{y}_{2 l}(s)+\right)}+C_{2}\left(s, \tilde{y}_{2 l}(s)+\right)\left(\left(s-\tilde{y}_{2 l}(s)\right)-\right)^{\gamma_{2}\left(s, \tilde{y}_{2 l}(s)+\right)} \\
& \quad=V\left(s-\tilde{y}_{2 l}(s), s, \tilde{y}_{2 l}(s) ; b\left(s, \tilde{y}_{2 l}(s)\right)\right) \\
& \quad C_{1}\left(s, \tilde{y}_{2 l-1}(s)\right)\left(s-\tilde{y}_{2 l-1}(s)\right)^{\gamma_{1}\left(s, \tilde{y}_{2 l-1}(s)\right)}+C_{2}\left(s, \tilde{y}_{2 l-1}(s)\right)\left(s-\tilde{y}_{2 l-1}(s)\right)^{\gamma_{2}\left(s, \tilde{y}_{2 l-1}(s)\right)} \\
& \quad=V\left(\left(s-\tilde{y}_{2 l-1}(s)\right)-, s, \tilde{y}_{2 l-1}(s)+; b_{*}\left(s, \tilde{y}_{2 l-1}(s)+\right)\right),
\end{aligned}
$$

for $s>K$ and $l \in \mathbb{N}$, where the right-hand sides are given by (41)-(42) with $b_{*}\left(s, \tilde{y}_{2 l-1}(s)+\right)=$ $b_{*}\left(s, \tilde{y}_{2 l}(s)\right)=s$. However, if $b_{*}(s, s-)=h_{*}(s)>s$ holds with $h_{*}(s)$ given by (31) then we 
have $\tilde{y}_{1}(s)=s-$ and condition (48) for $l=1$ changes its form to $C_{2}(s, s-)=0$ for $s>K$, since otherwise $V(x, s, s-) \rightarrow \pm \infty$ as $x \downarrow 0$, which must be excluded by virtue of the obvious fact that the value function in (4) is bounded at 0 .

In addition, the process $(X, S, Y)$ can exit the region $\widetilde{R}_{2 l}^{3}$ in (46) passing to the stopping region $D_{*}$ from (9) only through the point $(\bar{s}(y), \bar{s}(y), y)$, by hitting the plane $d_{1}^{3}$ and increasing its second component $S$ until it reaches the value $\bar{s}(y)=\inf \left\{q>s \mid b_{*}(q, y) \leq q\right\}$. Observe that the boundary $b_{*}(q, y)$ provides the unique solution of (43) with the starting value $b_{*}(q, q-)=h_{*}(q)$ for each $q \leq \bar{s}(y)$, given that this solution stays strictly above the surface $x=K \vee(r K / \delta(q, y))$. Then the candidate value function should be continuous at the point $(\bar{s}(y), \bar{s}(y), y)$, which is expressed by the equality

$$
\begin{aligned}
& C_{1}(\bar{s}(y)-, y)(\bar{s}(y)-)^{\gamma_{1}(\bar{s}(y)-, y)}+C_{2}(\bar{s}(y)-, y)(\bar{s}(y)-)^{\gamma_{2}(\bar{s}(y)-, y)} \\
& \quad=V\left(\bar{s}(y), \bar{s}(y), y ; b_{*}(\bar{s}(y), y)\right) \\
& \quad \equiv \bar{s}(y)-K
\end{aligned}
$$

for each $\tilde{y}_{2 l}(s)<y \leq \tilde{y}_{2 l-1}(s), l \in \mathbb{N}$, and $s>K$. We can therefore conclude that the candidate value function admits the representation

$$
\begin{aligned}
V\left(x, s, y ; \bar{s}(y), \tilde{y}_{2 l-1}(s), \tilde{y}_{2 l}(s)\right)= & C_{1}\left(s, y ; \bar{s}(y), \tilde{y}_{2 l-1}(s), \tilde{y}_{2 l}(s)\right) x^{\gamma_{1}(s, y)} \\
& +C_{2}\left(s, y ; \bar{s}(y), \tilde{y}_{2 l-1}(s), \tilde{y}_{2 l}(s)\right) x^{\gamma_{2}(s, y)}
\end{aligned}
$$

in the regions $\widetilde{R}_{2 l}^{3}$ given by (46), where $C_{i}\left(s, y ; \bar{s}(y), \tilde{y}_{2 l-1}(s), \tilde{y}_{2 l}(s)\right), i=1,2$, provide a unique solution of the two-dimensional system of first-order linear partial differential equations in (25)-(26) with boundary conditions (47)-(49) for $l \in \mathbb{N}$. Finally, we observe that the candidate value function should be given by the condition in the right-hand part of (12) in the regions

$$
\widehat{R}_{2 k}^{3}=\left\{(x, s, y) \in E^{3} \mid \widehat{y}_{2 k}(s)<y \leq \widehat{y}_{2 k-1}(s)\right\}
$$

for $k \in \mathbb{N}$, which belong to the stopping region $D_{*}$ in (9).

3.3.2. The put option case. Let us finally consider the payoff function $G(x)=(L-x)^{+}$in (4). In this case, solving the system of equations in (21) and (23) we conclude that the function in (19) admits the representation

$$
V\left(x, s, y ; a_{*}(s, y)\right)=C_{1}\left(s, y ; a_{*}(s, y)\right) x^{\gamma_{1}(s, y)}+C_{2}\left(s, y ; a_{*}(s, y)\right) x^{\gamma_{2}(s, y)}
$$

for $0<s-y \leq a_{*}(s, y)<x \leq s$, with

$$
C_{i}\left(s, y ; a_{*}(s, y)\right)=\frac{\left(\gamma_{3-i}(s, y)-1\right) a_{*}(s, y)-\gamma_{3-i}(s, y) L}{\left(\gamma_{i}(s, y)-\gamma_{3-i}(s, y)\right) a_{*}^{\gamma_{i}(s, y)}(s, y)}
$$

for all $0<y<s$ and $i=1,2$. Hence, assuming that the boundary function $a_{*}(s, y)$ is continuously differentiable, we apply condition (25) to the functions $C_{i}(s, y)=C_{i}\left(s, y ; a_{*}(s, y)\right)$, $i=1,2$, in (52) to conclude that $a_{*}(s, y)$ solves the first-order nonlinear ordinary differential equation

$$
\begin{aligned}
\partial_{s} a(s, y)=\sum_{i=1}^{2} & \frac{\left(\left(\gamma_{3-i}(s, y)-1\right) a(s, y)-\gamma_{3-i}(s, y)\right) a(s, y)}{\left(\gamma_{i}(s, y)-1\right)\left(\gamma_{3-i}(s, y)-1\right) a(s, y)-\gamma_{i}(s, y) \gamma_{3-i}(s, y) L} \\
& \times\left(\frac{1}{\gamma_{3-i}(s, y)-\gamma_{i}(s, y)}+\frac{(s / a(s, y))^{\gamma_{i}(s, y)} \ln (s / a(s, y))}{(s / a(s, y))^{\gamma_{i}(s, y)}-(s / a(s, y))^{\gamma_{3-i}(s, y)}}\right) \\
& \times \partial_{s} \gamma_{i}(s, y)
\end{aligned}
$$

for $0<y<s$, where the partial derivatives $\partial_{s} \gamma_{i}(s, y), i=1,2$, are given by (27) with (29). 
Since the functions $\delta(s, y)$ and $\sigma(s, y)$ are assumed to be continuously differentiable and bounded, the limits $\delta(y+, y)$ and $\sigma(y+, y)$ exist for each $y>0$. Then the limits $\gamma_{i}(y+, y)$ can be identified with the functions $\beta_{i}(y), i=1,2$, from Subsection 3.2, and the function in (51) should satisfy the property $V\left(x, s, y ; a_{*}(s, y)\right) \rightarrow V\left(x, y+, y ; a_{*}(y+, y)\right)$ as $s \downarrow y$ for each $s-y \leq a_{*}(s, y)<x \leq s$. Thus, we conclude that the equalities

$$
V\left(x, y+, y ; a_{*}(y+, y)\right)=U\left(x, y ; a_{*}(y+, y)\right) \quad \text { and } \quad a_{*}(y+, y)=g_{*}(y)
$$

hold for $0<a_{*}(y+, y)<x \leq y$ and $U\left(x, s ; g_{*}(s)\right)$ given by (36) with $g_{*}(s)$ obtained in Subsection 3.2.2. To see this, we observe that the candidate value function evaluated at $s \downarrow y$ in (54) satisfies the normal reflection condition only at the diagonal $d_{3}^{3}=\left\{(x, s, y) \in \mathbb{R}^{3} \mid 0<\right.$ $x=s=y\}$ of the plane $d_{1}^{3}$, and, thus, the function $a_{*}(y+, y)=g_{*}(y)$ is the maximal solution of (38) with the boundary condition $a_{*}(\infty, \infty)=g_{*}(\infty)$ of (39) as $y=s \rightarrow \infty$ and such that this solution stays strictly below the curve $x=L \wedge(r L / \delta(y))$.

For any $y>0$ fixed, let us now consider the unique solution $a_{*}(s, y)$ of (53) started at the value $a_{*}(y+, y)=g_{*}(y)$, given that this solution stays strictly below the surface $x=L \wedge(r L / \delta(s, y))$. Then we put $\widetilde{s}_{0}(y)=y$ and define an increasing sequence $\left(\widetilde{s}_{n}(y)\right)_{n \in \mathbb{N}}$ such that the boundary $a_{*}(s, y)$ exits the region $E^{3}$ from the side of the plane $d_{1}^{3}$ at the points $\left(\widetilde{s}_{2 l-1}(y), \widetilde{s}_{2 l-1}(y), y\right)$ and enters $E^{3}$ upwards at the points $\left(\widetilde{s}_{2 l}(y), \widetilde{s}_{2 l}(y), y\right)$. Namely, we define $\tilde{s}_{2 l-1}(y)=\inf \left\{s>\tilde{s}_{2 l-2}(y) \mid a_{*}(s, y)>s\right\}$ and $\tilde{s}_{2 l}(y)=\inf \left\{s>\tilde{s}_{2 l-1}(y) \mid a_{*}(s, y) \leq\right.$ $s\}, l \in \mathbb{N}$, whenever they exist, and put $\tilde{s}_{2 l-1}(y)=\tilde{s}_{2 l}(y)=\infty$ otherwise for $l \in \mathbb{N}$. Note that $y<\widetilde{s}_{2 l-1}(y)<\widetilde{s}_{2 l}(y) \leq L, l \in \mathbb{N}$, by construction. Moreover, we put $\widehat{s}_{0}(y)=y$ and define an increasing sequence $\left(\widehat{s}_{n}(y)\right)_{n \in \mathbb{N}}$ such that $\widehat{s}_{2 k-1}(y)=\inf \left\{s>\widehat{s}_{2 k-2}(y) \mid a_{*}(s, y)<s-y\right\}$ and $\widehat{s}_{2 k}(y)=\inf \left\{s>\widehat{s}_{2 k-1}(y) \mid a_{*}(s, y) \geq s-y\right\}, k \in \mathbb{N}$, whenever they exist, and put $\widehat{s}_{2 k-1}(y)=\widehat{s}_{2 k}(y)=\infty$ otherwise. Note that $y \leq \widehat{s}_{2 k-2}(y)<\widehat{s}_{2 k-1}(y)<L+y$, by construction, for $k=1, \ldots, \widehat{k}$, where $\widehat{k}=\sup \left\{k \in \overline{\mathbb{N}} \mid \widehat{s}_{2 k-1}(y)-y<L\right\}$. Therefore, the candidate value function admits the expression in (51) in either the region

$$
\widehat{Q}_{2 k-2}^{3}=\left\{(x, s, y) \in E^{3} \mid \widehat{s}_{2 k-2}(y) \leq s<\min _{l \in \mathbb{N}}\left\{\widetilde{s}_{2 l-1}(y) \mid \widetilde{s}_{2 l-1}(y)>\widehat{s}_{2 k-2}(y)\right\} \wedge \widehat{s}_{2 k-1}(y)\right\}
$$

or

$$
\widetilde{Q}_{2 l-2}^{3}=\left\{(x, s, y) \in E^{3} \mid \widetilde{s}_{2 l-2}(y) \leq y<\min _{k \in \mathbb{N}}\left\{\widehat{s}_{2 k-1}(y) \mid \widehat{s}_{2 k-1}(y)>\widetilde{s}_{2 l-2}(y)\right\} \wedge \widetilde{s}_{2 l-1}(y)\right\}
$$

for $k=1, \ldots, \widehat{k}$, and $l \in \mathbb{N}$, and the boundary function $a_{*}(s, y)$ provides the unique solution of (53) started at the value $a_{*}(y+, y)=g_{*}(y)$, given that this solution stays strictly below the surface $x=L \wedge(r L / \delta(s, y))$ (see Figure 3$)$.

On the other hand, the candidate value function takes the form of (19) with $C_{i}(s, y), i=1,2$, solving the linear system of first-order partial differential equations in (25) and (26) in the regions

$$
\widehat{Q}_{2 k-1}^{3}=\left\{(x, s, y) \in E^{3} \mid \widehat{s}_{2 k-1}(y) \leq s<\widehat{s}_{2 k}(y)\right\}
$$

for $k=1, \ldots, \widehat{k}$, which belong to $C^{\prime}$ in (7). Note that the process $(X, S, Y)$ can enter $\widehat{Q}_{2 k-1}^{3}$ in (57) from one of the regions $\widehat{Q}_{2 k-2}^{3}$ in (55) or $\widetilde{Q}_{2 l-2}^{3}$ in (56) for some $l \in \mathbb{N}$ only through the point $\left.\widehat{s}_{2 k-1}(y), \widehat{s}_{2 k-1}(y), y\right)$, and can exit $\widehat{Q}_{2 k-1}^{3}$ passing to $\widehat{Q}_{2 k}^{3}$ only through the point $\left(\widehat{s}_{2 k}(y), \widehat{s}_{2 k}(y), y\right)$ by hitting the plane $d_{1}^{3}$ and increasing its second component $S$. Thus, the candidate value function should be continuous at the points $\left.\widehat{s}_{2 k-1}(y), \widehat{s}_{2 k-1}(y), y\right)$ and 


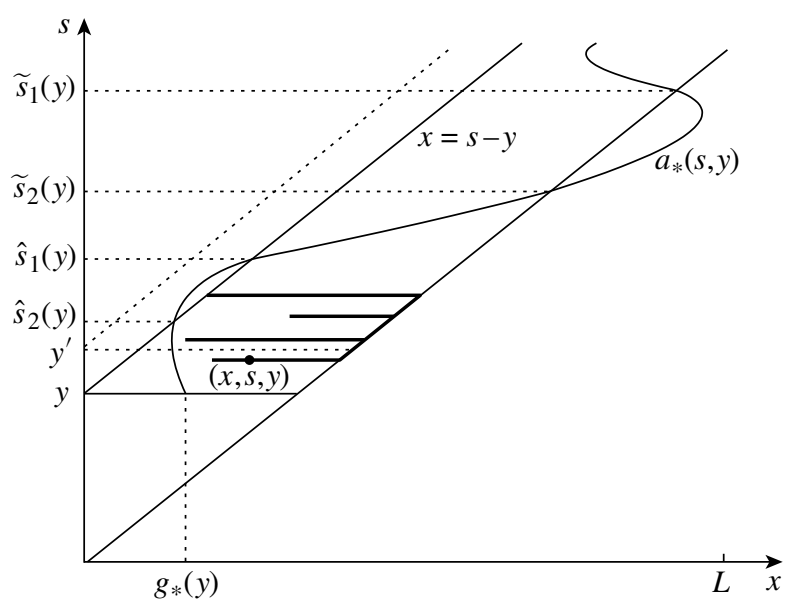

FiguRE 3: A computer drawing of the state space of the process $(X, S, Y)$ for some fixed $y$, which increases to $y^{\prime}$, and the boundary function $a_{*}(s, y)$.

$\left(\widehat{s}_{2 k}(y), \widehat{s}_{2 k}(y), y\right)$, which is expressed by the equalities

$$
\begin{aligned}
& C_{1}\left(\widehat{s}_{2 k-1}(y), y\right)\left(\widehat{s}_{2 k-1}(y)\right)^{\gamma_{1}\left(\widehat{s}_{2 k-1}(y), y\right)}+C_{2}\left(\widehat{s}_{2 k-1}(y), y\right)\left(\widehat{s}_{2 k-1}(y)\right)^{\left.\gamma_{2} \widehat{s}_{2 k-1}(y), y\right)} \\
& \quad=V\left(\widehat{s}_{2 k-1}(y)-, \widehat{s}_{2 k-1}(y)-, y ; a_{*}\left(\widehat{s}_{2 k-1}(y)-, y\right)\right) \\
& C_{1}\left(\widehat{s}_{2 k}(y)-, y\right)\left(\widehat{s}_{2 k}(y)-\right)^{\gamma_{1}\left(\widehat{s}_{2 k}(y)-, y\right)}+C_{2}\left(\widehat{s}_{2 k}(y)-, y\right)\left(\widehat{s}_{2 k}(y)-\right)^{\gamma_{2}\left(\widehat{s}_{2 k}(y)-, y\right)} \\
& \quad=V\left(\widehat{s}_{2 k}(y), \widehat{s}_{2 k}(y), y ; a_{*}\left(\widehat{s}_{2 k}(y), y\right)\right)
\end{aligned}
$$

for $y>0$ and $k=1, \ldots, \widehat{k}-1$, where the right-hand sides are given by (51)-(52) with $a_{*}\left(\widehat{s}_{2 k-1}(y)-, y\right)=\left(\widehat{s}_{2 k-1}(y)-y\right)-$ and $a_{*}\left(\widehat{s}_{2 k}(y), y\right)=\widehat{s}_{2 k}(y)-y$, respectively. Moreover, in the region $\widehat{Q}_{2 \widehat{k}-1}^{3}$ we have $\widehat{s}_{2 \widehat{k}}(y)=\infty$ and condition (59) for $k=\widehat{k}$ changes its form to $C_{1}(\infty, y)=0$ for $y>0$, since otherwise $V(x, \infty, y) \rightarrow \pm \infty$ as $x \uparrow \infty$, which must be excluded due to the fact that the value function in (4) is bounded at $\infty$, while condition (58) holds for $k=\widehat{k}$ as well.

In addition, the process $(X, S, Y)$ can exit $\widehat{Q}_{2 k-1}^{3}$ in (57), passing to the stopping region $D_{*}$ in (9) only through the point $(s-\bar{y}(s), s, \bar{y}(s))$ by hitting the plane $d_{2}^{3}$ and increasing its third component $Y$ until it reaches the value $\bar{y}(s)=\inf \left\{z>y \mid a_{*}(s, z) \geq s-z\right\}$. Observe that the boundary $a_{*}(s, z)$ provides the unique solution of (53) with the starting value $a_{*}(z+, z)=$ $g_{*}(z)$ from (38) for each $z<\bar{y}(s)$, given that this solution stays strictly below the surface $x=L \wedge(r L / \delta(s, z))$. Then the candidate value function should be continuous at the point $(s-\bar{y}(s), s, \bar{y}(s))$, which is expressed by the equality

$$
\begin{aligned}
C_{1}(s, & \bar{y}(s)-)((s-\bar{y}(s))+)^{\gamma_{1}(s, \bar{y}(s)-)}+C_{2}(s, \bar{y}(s)-)((s-\bar{y}(s))+)^{\gamma_{2}(s, \bar{y}(s)-)} \\
& =V\left(s-\bar{y}(s), s, \bar{y}(s) ; a_{*}(s, \bar{y}(s))\right) \\
& \equiv L-(s-\bar{y}(s))
\end{aligned}
$$

for each $\widehat{s}_{2 k-1}(y) \leq s<\widehat{s}_{2 k}(y), k=1, \ldots, \widehat{k}$, and $y>0$. We can therefore conclude that the candidate value function admits the representation

$$
\begin{aligned}
V\left(x, s, y ; \widehat{s}_{2 k-1}(y), \widehat{s}_{2 k}(y), \bar{y}(s)\right)= & C_{1}\left(s, y ; \widehat{s}_{2 k-1}(y), \widehat{s}_{2 k}(y), \bar{y}(s)\right) x^{\gamma_{1}(s, y)} \\
& +C_{2}\left(s, y ; \widehat{s}_{2 k-1}(y), \widehat{s}_{2 k}(y), \bar{y}(s)\right) x^{\gamma_{2}(s, y)}
\end{aligned}
$$


in the regions $\widehat{Q}_{2 k-1}^{3}$ in (57), where $C_{i}\left(s, y ; \widehat{s}_{2 k-1}(y), \widehat{s}_{2 k}(y), \bar{y}(s)\right), i=1,2$, provide a unique solution of the two-dimensional system of linear partial differential equations in (25)-(26) with the boundary conditions (58)-(60) for $k=1, \ldots, \widehat{k}$. Finally, we note that the candidate value function should be given by the condition in the left-hand part of (12) in the regions

$$
\widetilde{Q}_{2 l-1}^{3}=\left\{(x, s, y) \in E^{3} \mid \widetilde{s}_{2 l-1}(y) \leq s<\widetilde{s}_{2 l}(y)\right\}
$$

for $l \in \mathbb{N}$, which belong to the stopping region $D_{*}$ from (9).

\section{Main results and proof}

In this section, taking into account the facts proved above, we formulate and prove the main results of the paper. We recall that the process $(X, S, Y)$ is defined in (1)-(3).

Theorem 1. In the perpetual American call option case with payoff function $G(x)=(x-K)^{+}$, the value function of the optimal stopping problem (4) has the expression

$$
V_{*}(x, s, y)= \begin{cases}V\left(x, s, y ; b_{*}(s, y)\right) & \text { if } s-y \leq x<b_{*}(s, y) \leq s, \\ V\left(x, s, y ; \bar{s}(y), \tilde{y}_{2 l-1}(s), \tilde{y}_{2 l}(s)\right) & \text { if } s-y \leq x \leq s<b_{*}(s, y), \\ x-K & \text { if } b_{*}(s, y) \leq x \leq s,\end{cases}
$$

and the optimal stopping time is given by the right-hand part of (6), where the functions $V\left(x, s, y ; b_{*}(s, y)\right)$ and $V\left(x, s, y ; \bar{s}(y), \tilde{y}_{2 l-1}(s), \tilde{y}_{2 l}(s)\right)$ as well as the boundary $b_{*}(s, y)$ are specified as follows.

(i) In the particular case $\delta(s, y)=\delta(s)$ and $\sigma(s, y)=\sigma(s)$, the function $V(x, s, y$; $\left.b_{*}(s, y)\right)=U\left(x, s ; h_{*}(s)\right)$ and the boundary $b_{*}(s, y)=h_{*}(s)$ are given by (31) for $(x, s) \in \widetilde{R}_{2 l-1}^{2}$ defined in (32), and $V\left(x, s, y ; \bar{s}(y), \tilde{y}_{2 l-1}(s), \tilde{y}_{2 l}(s)\right)=U\left(x, s ; \tilde{s}_{2 l-1}\right)$ is given by (35) whenever $(x, s) \in \widetilde{R}_{2 l}^{2}$ defined in (33) for $l \in \mathbb{N}$.

(ii) In the general case for $\delta(s, y)$ and $\sigma(s, y)$, the function $V\left(x, s, y ; b_{*}(s, y)\right)$ is given by (41)-(42) and the boundary $b_{*}(s, y)$ provides the unique solution of (43) started at the value $b_{*}(s, s-)=h_{*}(s)$ from (31), given that $b_{*}(s, y)>K \vee(r K / \delta(s, y))$ holds for $(x, s, y) \in \widetilde{R}_{2 l-1}^{3} \cup \widehat{R}_{2 k-1}^{3}$ defined in (44) and (45), respectively, and $V(x, s, y$; $\left.\bar{s}(y), \tilde{y}_{2 l-1}(s), \tilde{y}_{2 l}(s)\right)$ is given by (50) whenever $(x, s, y) \in \widetilde{R}_{2 l}^{3}$ defined in (46) with $C_{i}\left(s, y ; \bar{s}(y), \tilde{y}_{2 l-1}(s), \tilde{y}_{2 l}(s)\right), i=1,2$, solving the system of equations in (25)-(26) and satisfying conditions (47)-(49) for $k, l \in \mathbb{N}$, where (48) changes its form to $C_{2}(s, s-)=0$ when $l=1$, if $b_{*}(s, s-)=h_{*}(s)>s$ holds.

Theorem 2. In the perpetual American put option case with payoff function $G(x)=(L-x)^{+}$, the value function of the optimal stopping problem (4) has the expression

$$
V_{*}(x, s, y)= \begin{cases}V\left(x, s, y ; a_{*}(s, y)\right) & \text { if } s-y \leq a_{*}(s, y)<x \leq s, \\ V\left(x, s, y ; \widehat{s}_{2 k-1}(y), \widehat{s}_{2 k}(y), \bar{y}(s)\right) & \text { if } a_{*}(s, y)<s-y \leq x \leq s, \\ L-x & \text { if } s-y \leq x \leq a_{*}(s, y),\end{cases}
$$

and the optimal stopping time is given by the left-hand part of (6), where the functions $V\left(x, s, y ; a_{*}(s, y)\right)$ and $V\left(x, s, y ; \widetilde{s}_{2 l-1}(y), \widetilde{s}_{2 l}(y), \bar{y}(s)\right)$ as well as the boundary $a_{*}(s, y)$ are specified as follows. 
(i) In the particular case $\delta(s, y)=\delta(s)$ and $\sigma(s, y)=\sigma(s)$, the function $V(x, s, y$; $\left.a_{*}(s, y)\right)=U\left(x, s ; g_{*}(s)\right)$ is given by (36)-(37) and the boundary $a_{*}(s, y)=g_{*}(s)$ provides the maximal solution of (38) started at $g_{*}(\infty)$ from (39) such that $g_{*}(s)<$ $L \wedge(r L / \delta(s))$ holds for $(x, s) \in \widehat{Q}_{2 k-1}^{2}$ defined in $(40)$ and $k \in \mathbb{N}$.

(ii) In the general case for $\delta(s, y)$ and $\sigma(s, y)$, the function $V\left(x, s, y ; a_{*}(s, y)\right)$ is given by $(51)-(52)$ and the boundary $a_{*}(s, y)$ provides the unique solution of (53) started from the value $a_{*}(y+, y)=g_{*}(y)$ from part $(i)$, given that $a_{*}(s, y)<L \wedge(r L / \delta(s, y))$ holds for $(x, s, y) \in \widehat{Q}_{2 k-2}^{3} \cup \widetilde{Q}_{2 l-2}^{3}$ defined in (55) and (56), respectively, and $V(x, s, y$; $\left.\widehat{s}_{2 k-1}(y), \widehat{s}_{2 k}(y), \bar{y}(s)\right)$ is given by (61) whenever $(x, s, y) \in \widehat{Q}_{2 k-1}^{3}$ defined in (57) with $C_{i}\left(s, y ; \widehat{s}_{2 k-1}(y), \widehat{s}_{2 k}(y), \bar{y}(s)\right), i=1,2$, solving the system of equations in (25)-(26) and satisfying conditions (58)-(60) for $k=1, \ldots, \widehat{k}$ and $l \in \mathbb{N}$, where (59) changes its form to $C_{1}(\infty, y)=0$ when $k=\widehat{k}$.

Since all the parts of the assertions formulated above are proved using similar arguments, we only give a proof for the three-dimensional optimal stopping problem related to the perpetual American put option in part (ii) of Theorem 2, which represents the most complicated and informative case.

Proof of Theorem 2. In order to verify the assertion, it remains to show that the function defined in (62) coincides with the value function in (4) and that the stopping time $\tau_{*}$ in the left-hand part of (6) is optimal with the boundary $a_{*}(s, y)$ specified above. For this, let $a(s, y)$ be the unique solution of (53) starting from the value $a(y+, y)=g(y)$, being any solution of (38) starting from $a_{*}(\infty, \infty)=g_{*}(\infty)$ in (39) and satisfying $g(y)<L \wedge(r L / \delta(y))$ for all $y>0$. Let us also denote by $V_{a}(x, s, y)$ the right-hand side of (62) associated with this $a(s, y)$. It then follows using straightforward calculations and the assumptions presented above that the function $V_{a}(x, s, y)$ solves the left-hand system of (10)-(12), while the normal reflection and smooth-fit conditions satisfy (15) and the left-hand part of (16). Hence, taking into account the fact that the function $V_{a}(x, s, y)$ is $C^{2,1,1}$ and the boundary $a(s, y)$ is assumed to be continuously differentiable for all $0<y<s$, by applying the change-of-variable formula from [23, Theorem 3.1] to $\mathrm{e}^{-r t} V_{a}\left(X_{t}, S_{t}, Y_{t}\right)$, we obtain

$$
\begin{aligned}
\mathrm{e}^{-r t} V_{a}\left(X_{t}, S_{t}, Y_{t}\right)= & V_{a}(x, s, y)+M_{t} \\
& +\int_{0}^{t} \mathrm{e}^{-r u}\left(\mathbb{L} V_{a}-r V_{a}\right)\left(X_{u}, S_{u}, Y_{u}\right) \\
& \quad \times I\left(X_{u} \neq S_{u}-Y_{u}, X_{u} \neq a\left(S_{u}, Y_{u}\right), X_{u} \neq S_{u}\right) \mathrm{d} u \\
& +\int_{0}^{t} \mathrm{e}^{-r u} \partial_{s} V_{a}\left(X_{u}, S_{u}, Y_{u}\right) I\left(X_{u}=S_{u}\right) \mathrm{d} S_{u} \\
& +\int_{0}^{t} \mathrm{e}^{-r u} \partial_{y} V_{a}\left(X_{u}, S_{u}, Y_{u}\right) I\left(X_{u}=S_{u}-Y_{u}\right) \mathrm{d} Y_{u},
\end{aligned}
$$

where $I(\cdot)$ denotes the indicator function and the process $M=\left(M_{t}\right)_{t \geq 0}$, given by

$$
M_{t}=\int_{0}^{t} \mathrm{e}^{-r u} \partial_{x} V_{a}\left(X_{u}, S_{u}, Y_{u}\right) I\left(X_{u} \neq S_{u}-Y_{u}, X_{u} \neq S_{u}\right) \sigma\left(S_{u}, Y_{u}\right) X_{u} \mathrm{~d} B_{u},
$$

is a square-integrable martingale under $\mathbb{P}_{x, s, y}$. Note that since the time spent by the process $X$ at the boundary surface $\left\{(x, s, y) \in E^{3} \mid x=a(s, y)\right\}$ as well as at the planes $d_{1}^{3}$ and $d_{2}^{3}$ is of Lebesgue measure 0 , the indicators in the second line of (63) as well as in (64) can be ignored. 
Moreover, since the process $S$ increases only at the plane $d_{1}^{3}$ and the process $Y$ increases only at the plane $d_{2}^{3}$, the indicators in the third line of (63) can also be set equal to 1 .

By using straightforward calculations and the arguments from the previous section, it is verified that $\left(\mathbb{L} V_{a}-r V_{a}\right)(x, s, y) \leq 0$ for all $(x, s, y) \in E^{3}$ such that $x \neq a(s, y), x \neq s-y$, and $x \neq s$. Moreover, it is shown by means of standard arguments that the property in the left-hand part of (13) also holds, which together with the left-hand parts of (11)-(12) implies that the inequality $V_{a}(x, s, y) \geq(L-x)^{+}$is satisfied for all $(x, s, y) \in E^{3}$. It therefore follows from (63) that the inequalities

$$
\mathrm{e}^{-r \tau}\left(L-X_{\tau}\right)^{+} \leq \mathrm{e}^{-r \tau} V_{a}\left(X_{\tau}, S_{\tau}, Y_{\tau}\right) \leq V_{a}(x, s, y)+M_{\tau}
$$

hold for any finite stopping time $\tau$ with respect to the natural filtration of $X$.

Taking the expectation with respect to $\mathbb{P}_{x, s, y}$ in (65) by means of the optional sampling theorem (see, e.g. [16, Chapter I, Theorem 3.22]), we obtain

$$
\begin{aligned}
\mathbb{E}_{x, s, y}\left[\mathrm{e}^{-r(\tau \wedge t)}\left(L-X_{\tau \wedge t}\right)^{+}\right] & \leq \mathbb{E}_{x, s, y}\left[\mathrm{e}^{-r(\tau \wedge t)} V_{a}\left(X_{\tau \wedge t}, S_{\tau \wedge t}, Y_{\tau \wedge t}\right)\right] \\
& \leq V_{a}(x, s, y)+\mathbb{E}_{x, s, y} M_{\tau \wedge t} \\
& =V_{a}(x, s, y)
\end{aligned}
$$

for all ( $x, s, y) \in E^{3}$. Hence, letting $t$ go to $\infty$ and using Fatou's lemma, we conclude that, for any finite stopping time $\tau$, the inequalities

$$
\mathbb{E}_{x, s, y}\left[\mathrm{e}^{-r \tau}\left(L-X_{\tau}\right)^{+}\right] \leq \mathbb{E}_{x, s, y}\left[\mathrm{e}^{-r \tau} V_{a}\left(X_{\tau}, S_{\tau}, Y_{\tau}\right)\right] \leq V_{a}(x, s, y)
$$

are satisfied for all $(x, s, y) \in E^{3}$. Taking first the supremum over all stopping times $\tau$ and then the infimum over all $a$, we conclude that

$$
\mathbb{E}_{x, s, y}\left[\mathrm{e}^{-r \tau_{*}}\left(L-X_{\tau_{*}}\right)^{+}\right] \leq \inf _{a} V_{a}(x, s, y)=V_{a_{*}}(x, s, y)
$$

where $a_{*}(s, y)$ is the unique solution of (53) starting from the value $a_{*}(y+, y)=g_{*}(y)$, which is the maximal solution to (38) being started at $a_{*}(\infty, \infty)=g_{*}(\infty)$ in (39) and staying strictly below the curve $x=L \wedge(r L / \delta(y))$. Using the fact that $V_{a}(x, s, y)$ is decreasing in the function $a<L \wedge(r L / \delta)$, we see that the infimum in (68) is attained over any sequence of solutions $\left(a_{n}(s, y)\right)_{n \in \mathbb{N}}$ to (53) started at the values $a_{n}(y+, y)=g_{n}(y)$, solving (38) and such that $g_{n}(y) \uparrow g_{*}(y)$, and, thus, $a_{n}(s, y) \uparrow a_{*}(s, y)$ as $n \rightarrow \infty$. Since the inequalities in (67) also hold for $a_{*}(s, y)$, we see that (68) holds for $a_{*}(s, y)$ and $(x, s, y) \in E^{3}$ as well. Note that $V_{a}(x, s, y)$ in (66) is superharmonic for the Markov process $(X, S, Y)$ on $E^{3}$. Taking into account the facts that $V_{a}(x, s, y)$ is decreasing in $a<L \wedge(r L / \delta)$ and the inequality $V_{a}(x, s, y) \geq(L-x)^{+}$ holds for all $(x, s, y) \in E^{3}$, we observe that the selection of the maximal solution $a_{*}(s, y)$, which stays strictly below the surface $x=L \wedge(r L / \delta(s, y))$ whenever such a choice exists, is equivalent to invoking the superharmonic characterization of the value function, which consists of constructing the smallest superharmonic function dominating the payoff function (see, e.g. [22] or [26, Chapter 1]).

In order to prove that $a_{*}(s, y)$ is optimal on $E^{3}$, we consider the sequence of stopping times $\tau_{n}$ defined as in the left-hand part of (6) with $a_{n}(s, y)$ instead of $a_{*}(s, y)$, where $a_{n}(s, y)$ is the unique solution of (53) started from the value $a_{n}(y+, y)=g_{n}(y)$ which solves (38) and starts at $a_{*}(\infty, \infty)=g_{*}(\infty)$ in (39), and such that $g_{n}\left(y_{n}\right)=L \wedge\left(r L / \delta\left(y_{n}\right)\right)$ for some $y_{n} \downarrow c$ as $n \rightarrow \infty$, where $c>0$ such that $g_{\infty}(y)<L \wedge(r L / \delta(y))$ for all $y \leq c$. By virtue of the 
fact that the function $V_{a_{n}}(x, s, y)$ from the right-hand side of (62) associated with this $a_{n}(s, y)$ satisfies the left-hand system of (10)-(13) with (15), and taking into account the structure of $\tau_{n}$ given by the left-hand part of (6) with $a_{n}(s, y)$ instead of $a_{*}(s, y)$, it follows from the equivalent expression of (63) that the equalities

$$
\mathrm{e}^{-r\left(\tau_{n} \wedge t\right)}\left(L-X_{\tau_{n} \wedge t}\right)^{+}=\mathrm{e}^{-r\left(\tau_{n} \wedge t\right)} V_{a_{n}}\left(X_{\tau_{n} \wedge t}, S_{\tau_{n} \wedge t}, Y_{\tau_{n} \wedge t}\right)=V_{a_{n}}(x, s, y)+M_{\tau_{n} \wedge t}
$$

hold for all $(x, s, y) \in E^{3}$. Observe that $\tau_{n} \uparrow \tau_{*}\left(\mathbb{P}_{x, s, y}\right.$-almost surely) and the variable $\mathrm{e}^{-r \tau_{*}}\left(L-X_{\tau_{*}}\right)^{+}$is bounded on the set $\left\{\tau_{*}=\infty\right\}$. Taking into account the fact that the boundary $a_{*}(s, y)$ is bounded, it is easily seen that the property $\mathbb{P}_{x, s, y}\left(\tau_{*}<\infty\right)=1$ holds for all $(x, s, y) \in E^{3}$. Hence, letting $t$ and $n$ go to $\infty$ and using the conditions in the left-hand part of (11) and (15), as well as the fact that $\tau_{n} \uparrow \tau_{*}\left(\mathbb{P}_{x, s, y}\right.$-almost surely), we can apply the Lebesgue dominated convergence theorem to (69) to obtain the equality

$$
\mathbb{E}_{x, s, y}\left[\mathrm{e}^{-r \tau_{*}}\left(L-X_{\tau_{*}}\right)^{+}\right]=V_{a_{*}}(x, s, y)
$$

for all $(x, s, y) \in E^{3}$, which together with (68) directly implies the desired assertion.

\section{Acknowledgements}

The authors are grateful to the anonymous referee for pointing out important issues and thereby improving the presentation of the paper. The first author gratefully acknowledges the fellowship for experienced researchers of the Alexander von Humboldt Foundation. The second author gratefully acknowledges the scholarship of the Alexander Onassis Public Benefit Foundation for his doctoral studies at the London School of Economics and Political Science. This paper was finished when the authors were visiting the Hausdorff Research Institute for Mathematics at the University of Bonn in the framework of the Trimester Program Stochastic Dynamics in Economics and Finance. The authors are thankful to the organisers of the programme for creating a unique atmosphere in a perfect research environment.

\section{References}

[1] Asmussen, S., Avram, F. And Pistorius, M. (2004). Russian and American put options under exponential phase-type Lévy models. Stoch. Process. Appl. 109, 79-111.

[2] Avram, F., Kyprianou, A. E. and Pistorius, M. R. (2004). Exit problems for spectrally negative Lévy processes and applications to (Canadized) Russian options. Ann. Appl. Prob. 14, 215-238.

[3] Baurdoux, E. J. And Kyprianou, A. E. (2009). The Shepp-Shiryaev stochastic game driven by a spectrally negative Lévy process. Theory Prob. Appl. 53, 481-499.

[4] Detemple, J. (2006). American-Style Derivatives: Valuation and Computation. Chapman and Hall/CRC, Boca Raton, FL.

[5] Dubins, L. E., Shepr, L. A. And Shiryaev, A. N. (1993). Optimal stopping rules and maximal inequalities for Bessel processes. Theory Prob. Appl. 38, 226-261.

[6] Dynkin, E. B. (1963). The optimum choice of the instant for stopping a Markov process. Soviet Math. Dokl. 4, 627-629.

[7] Forde, M. (2011). A diffusion-type process with a given joint law for the terminal level and supremum at an independent exponential time. Stoch. Process. Appl. 121, 2802-2817.

[8] Forde, M., Pogudin, A. And Zhang, H. (2013). Hitting times, occupation times, trivariate laws and the forward Kolmogorov equation for a one-dimensional diffusion with memory. Adv. Appl. Prob. 45, 860-875.

[9] GapeEv, P. V. (2007). Discounted optimal stopping for maxima of some jump-diffusion processes. J. Appl. Prob. 44, 713-731.

[10] Glover, K., Hulley, H. And Peskir, G. (2013). Three-dimensional Brownian motion and the golden ratio rule. Ann. Appl. Prob. 23, 895-922.

[11] Graversen, S. E. And Peskir, G. (1998). Optimal stopping and maximal inequalities for geometric Brownian motion. J. Appl. Prob. 35, 856-872. 
[12] Graversen, S. E. and PeskiR, G. (1998). Optimal stopping and maximal inequalities for linear diffusions. J. Theoret. Prob. 11, 259-277.

[13] Guo, X. AND Shepp, L. (2001). Some optimal stopping problems with nontrivial boundaries for pricing exotic options. J. Appl. Prob. 38, 647-658.

[14] Guo, X. And Zervos, M. (2010). $\pi$ options. Stoch. Process. Appl. 120, 1033-1059.

[15] JACKA, S. D. (1991). Optimal stopping and best constants for Doob-like inequalities. I. The case $p=1$. Ann. Prob. 19, 1798-1821.

[16] Karatzas, I. And Shreve, S. E. (1991). Brownian Motion and Stochastic Calculus, 2nd edn. Springer, New York.

[17] Liptser, R. S. And Shiryaev, A. N. (2001). Statistics of Random Processes I, 2nd edn. Springer, Berlin.

[18] Mijatović, A. AND Pistorius, M. R. (2012). On the drawdown of completely asymmetric Lévy processes. Stoch. Process. Appl. 122, 3812-3836.

[19] Отт, C. (2013). Optimal stopping problems for the maximum process with upper and lower caps. Ann. Appl. Prob. 23, 2327-2356.

[20] Отт, C. (2013). Optimal stopping problems for the maximum process. Doctoral Thesis, University of Bath.

[21] Pedersen, J. L. (2000). Discounted optimal stopping problems for the maximum process. J. Appl. Prob. 37, 972-983.

[22] Peskir, G. (1998). Optimal stopping of the maximum process: the maximality principle. Ann. Prob. 26, 16141640.

[23] Peskir, G. (2007). A change-of-variable formula with local time on surfaces. In Séminaire de Probababilités XL (Lecture Notes Math. 1899), Springer, Berlin, pp. 69-96.

[24] Peskir, G. (2012). Optimal detection of a hidden target: the median rule. Stoch. Process. Appl. 122, $2249-2263$.

[25] Peskir, G. (2014). Quickest detection of a hidden target and extremal surfaces. To appear in Ann. Appl. Prob.

[26] Peskir, G. And Shiryaev, A. (2006). Optimal Stopping and Free-Boundary Problems. Birkhäuser, Basel.

[27] Pospisil L., Vecer, J. and Hadjiliadis, O. (2009) Formulas for stopped diffusion processes with stopping times based on drawdowns and drawups. Stoch. Process. Appl. 119, 2563-2578.

[28] Shepp, L. and Shiryaev, A. N. (1993). The Russian option: reduced regret. Ann. Appl. Prob. 3, 631-640.

[29] Shepp, L. A. And Shiryaev, A. N. (1994). A new look at the 'Russian option'. Theory Prob. Appl. 39, $103-119$.

[30] Shiryaev, A. N. (1999). Essentials of Stochastic Finance. World Scientific, River Edge, NJ. 